\title{
Comparison of four phaC genes from Haloferax mediterranei and their function in different PHBV copolymer biosyntheses in Haloarcula hispanica
}

\author{
Jing Han', Ming Li', Jing Hou', Linping $\mathrm{Wu}^{2}$, Jian Zhou', Hua Xiang ${ }^{1 *}$
}

\begin{abstract}
Background: The halophilic archaeon Haloferax mediterranei is able to accumulate large amounts of poly(3hydroxybutyrate-co-3-hydroxyvalerate) (PHBV) with high molar fraction of 3-hydroxyvalerate (3HV) from unrelated carbon sources. A Polyhydroxyalkanoate (PHA) synthase composed of two subunits, $\mathrm{PhaC}_{\mathrm{Hme}}$ and $\mathrm{PhaE}_{\mathrm{Hme}}$, has been identified in this strain, and shown to account for the PHBV biosynthesis.

Results: With the aid of the genome sequence of Hfx. mediterranei CGMCC 1.2087, three additional phaC genes (designated phaC1, phaC2, and phaC3) were identified, which encoded putative PhaCs. Like PhaC $\mathrm{Hme}_{\text {( }}(54.8 \mathrm{kDa})$, PhaC1 (49.7 kDa) and PhaC3 (62.5 kDa) possessed the conserved motifs of type III PHA synthase, which was not observed in PhaC2 (40.4 kDa). Furthermore, the longer $C$ terminus found in the other three PhaCs was also absent

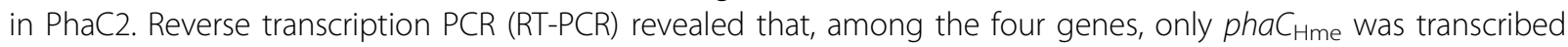
under PHA-accumulating conditions in the wild-type strain. However, heterologous coexpression of phaE $\mathrm{Hme}_{\mathrm{m} \text { with }}$ each phaC gene in Haloarcula hispanica PHB-1 showed that all PhaCs, except PhaC2, could lead to PHBV accumulation with various $3 \mathrm{HV}$ fractions. The three kinds of copolymers were characterized using gel-permeation chromatography (GPC), differential scanning calorimetry (DSC), and thermogravimetric analysis (TGA). Their thermal properties changed with the variations in monomer composition as well as the different molecular weights $\left(M_{\mathrm{w}}\right)$, thus might meet various application requirements.

Conclusion: We discover three cryptic phaC genes in Hfx. mediterranei, and demonstrate that genetic engineering of these newly identified phaC genes has biotechnological potential for PHBV production with tailor-made material properties.
\end{abstract}

\section{Background}

Polyhydroxyalkanoates (PHAs) are polymers of hydroxyalkanoates and are synthesized by various bacteria and some haloarchaeal strains [1]. The molecular weight of PHAs is generally distributed in the range of 50,000$1,000,000 \mathrm{Da}$, which is sufficient to provide these polymers with similar characteristics as conventional plastics [2]. However, due to the high production cost of PHAs, semi-commercial production has been realized only for the two common polyesters, poly(3-hydroxybutyrate) (PHB) and poly(3-hydroxybutyrate-co-3-hydroxyvalerate)

\footnotetext{
* Correspondence: xiangh@sun.im.ac.cn

'State Key Laboratory of Microbial Resources, Institute of Microbiology, Chinese Academy of Sciences, No.1 West Beichen Road, Chaoyang District, Beijing 100101, China

Full list of author information is available at the end of the article
}

(PHBV) [3-5]. Recently, halophilic Archaea have been attracting strong interest in PHBV research due to their comparable advantages as PHA producers, including utilizing unrelated cheap carbon sources, non-strict sterilization and easy method for PHA extraction [6-8]. A few haloarchaeal strains have been found to synthesize intracellular short-chain-length PHAs (scl-PHAs), PHB and PHBV, to serve as carbon and energy storage [8-16]. Compared with PHB, PHBV possesses better polymer properties and thus has wider applications in commodity materials [17-19]. Among the halophilic archaea, Haloferax mediterranei is one of the best-studied strains due to its capability to accumulate large amounts of PHBV from inexpensive carbon sources, including glucose, starch, and whey $[6-8,20,21]$. Therefore, it has 
been evaluated as one of the most promising candidate prokaryotes for the industrial production of PHBV [7].

PHA synthases are the key enzymes of PHA synthesis and catalyze the incorporation of 3-hydroxyacyl-CoA into PHAs. Over the past few decades, extensive research has been devoted to the study of PHA synthases in the domain of Bacteria. This enzyme is grouped into four types in bacteria, according to its substrate specificity and subunit composition [22]. The typical bacterial type III synthase is composed of two subunits, PhaE ( 40 kDa) and PhaC ( 40 kDa) [23]. In the domain of Archaea, a novel PHA synthase homologous to the bacterial type III PHA synthase, with subunits designated $\mathrm{PhaC}_{\mathrm{Hme}}$ and $\mathrm{PhaE}_{\mathrm{Hme}}$, has been identified and characterized in $H f x$. mediterranei [6]. As in bacteria, the PHA synthase-encoding genes (phaEC $C_{\mathrm{Hme}}$ ) in $H f x$. mediterranei are clustered and cotranscribed, and both $\mathrm{PhaC}_{\mathrm{Hme}}$ and $\mathrm{PhaE}_{\mathrm{Hme}}$ are indispensable for the PHBV synthesis from multiple unrelated carbon sources. The haloarchaeal PHA synthase and bacterial type III synthase share some conserved residues (e.g., the Cys-Asp-His catalytic triad). However, they are quite different in molecular weights and several characteristic motifs. The $\mathrm{PhaC}_{\mathrm{Hme}}(54.8$ $\mathrm{kDa})$ is much larger than its bacterial counterparts, in which the longer sequence at the $C$ terminus has been shown to be indispensable for enzyme activity [6]. In contrast, the PhaE $\mathrm{E}_{\mathrm{Hme}}(\sim 20 \mathrm{kDa})$ subunit is much smaller than its bacterial counterparts. Moreover, although "PhaE box" is strongly conserved in PhaEs of bacteria, it is missing in $\mathrm{PhaE}_{\mathrm{Hme}}$ of $H f x$. mediterranei.

Recently, the genome sequence of Hfx. mediterranei CGMCC 1.2087 has been completed in our laboratory (Han J. et al: Bioplastic production in the domain of Archaea: insights from the genome sequence of Haloferax mediterranei, in preparation). Intriguingly, sequence analysis revealed that three additional phaC paralogs (named phaC1, phaC2, and phaC3) were present and scattered throughout the whole genome. In this study, we compared the features of $\mathrm{PhaC}_{\mathrm{Hme}}$ and these three putative $\mathrm{PhaC}$ proteins (PhaC1-PhaC3), and determined their capabilities of PHA polymerizing in Haloarcula hispanica. Furthermore, the material properties of the produced polymers were also characterized using multiple methods.

\section{Results \\ Identification and analysis of the phaC genes in Hfx. mediterranei}

Hfx. mediterranei is comprised of one chromosome and three extrachromosomal elements (megaplasmids) [24]. The genome sequence of $H f x$. mediterranei CGMCC 1.2087 has been recently completed by our group. The genes encoding its PHA synthase, named phaE $E_{\mathrm{Hme}}$ and pha $C_{\mathrm{Hme}}$, have already been cloned and characterized [6] and were found to be located on megaplasmid pHM300. Interestingly, analysis of the whole genome sequence revealed that three other phaC paralogs existed in this strain and were designated phaC1, phaC2, and phaC3.

The phaC1 was mapped to the chromosome, whereas phaC2 and phaC3 were both mapped to megaplasmid pHM500 (Figure 1). Most of the genes adjacent to the four phaCs encoded conserved hypothetical proteins with unknown functions (Figure 1). The four PhaC proteins of $H f x$. mediterranei exhibited homology to each other (43\%-58\% identity), and they all contained the catalytic triad residues (Cys-Asp-His) (Figure 2). Both the "Lipase box-like" sequence (Gly-X-Cys-X-Gly-Gly) and the conserved motif of type III PHA synthase were present in $\mathrm{PhaC}_{\mathrm{Hme}}, \mathrm{PhaC} 1$, and $\mathrm{PhaC} 3$ (Figure 2). In the case of PhaC2, the last "Gly" residue of the "Lipase boxlike" sequence was replaced by an "Ala", and the conserved motif of the type III PHA synthase was also not strongly conserved (Figure 2). The deduced molecular weights of the four PhaCs were as follows: $\mathrm{PhaC}_{\mathrm{Hme}}{ }^{-}$ 54.8 kDa, PhaC1-49.7 kDa, PhaC2-40.4 kDa, and PhaC3-62.5 kDa. Only the molecular weight of $\mathrm{PhaC}_{\mathrm{Hme}}$ was equivalent to that of the PhaCs from Haloarcula marismortui (53.1 kDa; Accession no., YP_137339), Haloarcula hispanica (53.0 kDa; Accession no., ABV71394), and Haloquadratum walsbyi (52.1 kDa, Accession no., YP_658052). When compared with the primary sequences of the other three PhaCs, the $C$ terminal sequence was missing in $\mathrm{PhaC} 2$, which resulted in the smallest molecular weight.

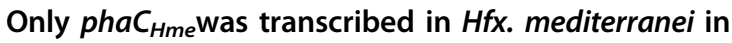 laboratory conditions}

In previous work, we found that the phaEC $C_{\mathrm{Hme}}$-deleted strain $H f x$. mediterranei PHBV-1 could not accumulate detectable scl-PHAs and expression of $p h a E_{\mathrm{Hme}}$ alone could not restore the ability to accumulate scl-PHAs [6], which indicated that there were no additional functional PhaC subunits in $H f x$. mediterranei $\mathrm{PHBV}-1$. To provide some clues to this phenomenon, RT-PCR with gene-specific primer pairs for the four phaC genes (Table 1) was employed to determine whether they were transcribed in the wild-type strain. The lengths of the PCR products of phaC $C_{\mathrm{Hme}}$, phaC1, phaC2, and phaC3 were $382 \mathrm{bp}$, $391 \mathrm{bp}, 408 \mathrm{bp}$, and $412 \mathrm{bp}$, respectively. The results demonstrated that of the four genes, only pha $C_{\mathrm{Hme}}$ was actively transcribed in MG medium (Figure 3A), which was consistent with our previous observation that $\mathrm{PhaC}_{\mathrm{Hme}}$ was indispensable for PHBV biosynthesis in $H f x$. mediterranei, as the other three phaCs were not expressed. 


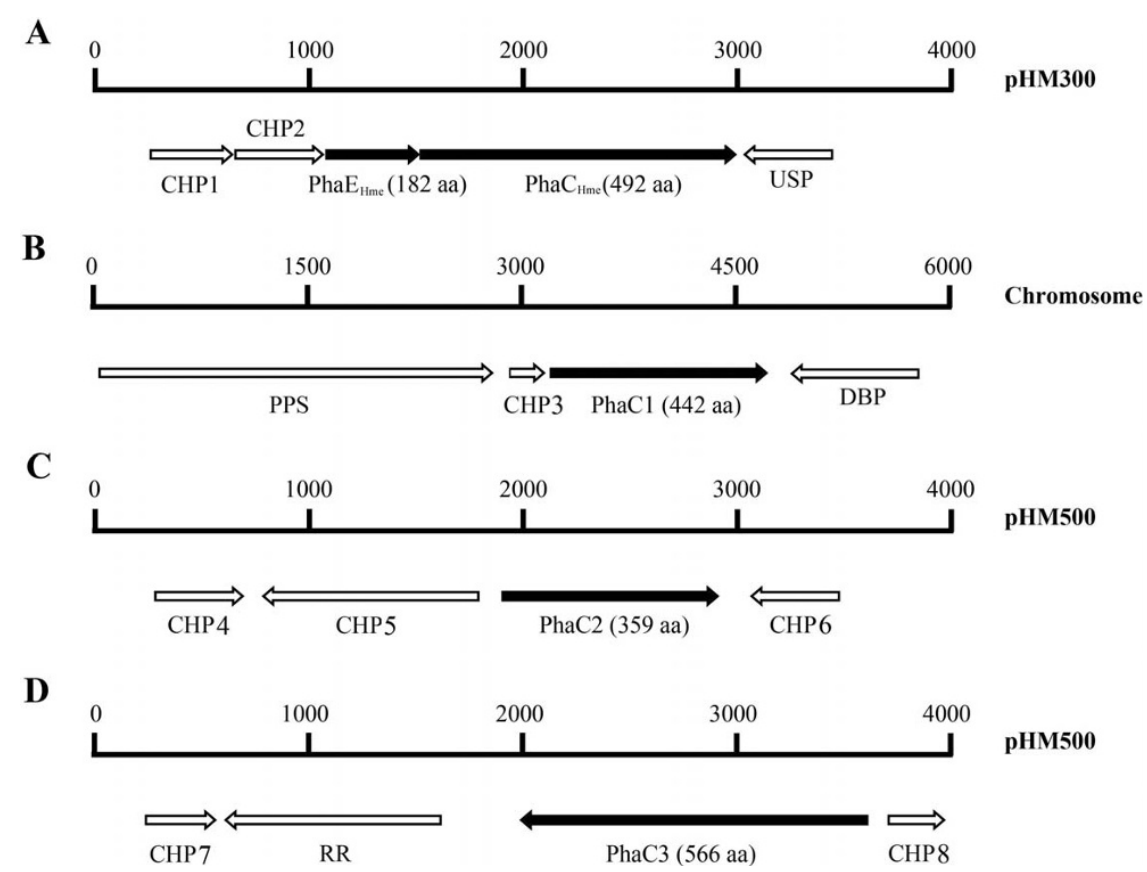

Figure 1 Schematic overview of the four phaC genes with their adjacent genes in $\boldsymbol{H f x}$. mediterranei. A: PhaEC $\mathrm{C}_{\text {me }}$ encoded by pHM300; $\mathrm{B}$ : PhaC1 encoded by the chromosome; C: PhaC2 encoded by pHM500; D: PhaC3 encoded by pHM500. The genes are shown as arrows. The numeric scale above the genes gives the length of the DNA (bp). The PHA synthase-encoding genes are shown in black. CHP: conserved hypothetical protein; DBP: DNA binding protein; PPS: phosphoenolpyruvate synthase; RR: Response regulator; USP: universal stress protein.

\section{Genetic analysis of the functions of the four PhaCs in}

\section{Haloarcula}

To determine if phaC1, phaC2, and phaC3 could encode functional proteins, a PHA synthase genedeleted strain, named Har. hispanica PHB-1 [13], was used to heterologously express these proteins. Because $\mathrm{PhaC}$ proteins were proposed to be functional combined with the PhaE subunit, the three expression plasmids designated pWL3EC1, pWL3EC2, and pWL3EC3 (Table 1) were constructed based on the $\mathrm{PhaE}_{\mathrm{Hme}}{ }^{-}$ expression plasmid pWL3E [6] and individually introduced into Har. hispanica PHB-1. During fermentation, Har. hispanica PHB-1/pWL3E [6] and the wild-type strain were used as the negative and positive control,

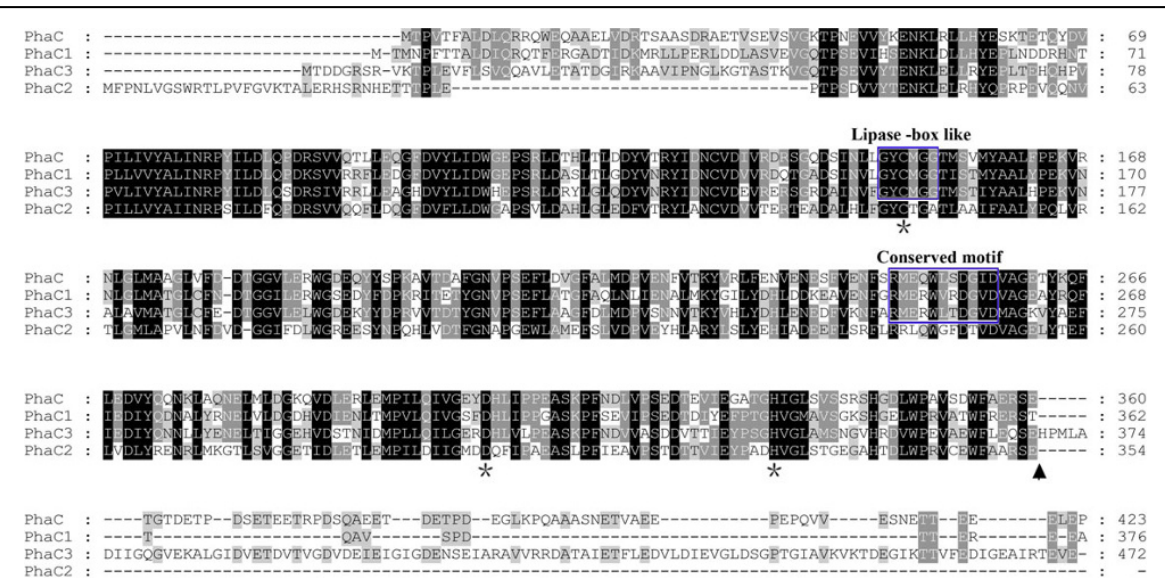

Figure 2 Multiple alignments of the amino acid sequences of four PhaCs from Hfx. mediterranei. The "Lipase box-like" and highly conserved motif of the Type III synthase are indicated. The conserved catalytic triad residues are indicated with asterisks. The position indicated with an arrow represents the $\mathrm{C}$ terminus sequence missing in PhaC2. Amino acids are given in standard one-letter abbreviations and the numbers indicate the positions of the amino acids within the respective proteins. Shaded amino acids residues indicate identical and conserved residues in the sequences of the four PhaCs. 
Table 1 Plasmids and primers used in this study

\begin{tabular}{lll}
\hline Plasmids or primers & Relevant characteristics & Sources or references \\
\hline Plasmids & & \\
pWL3E & $11.2-k b$, phaE $E_{H m e}$ and its native promoter & {$[6]$} \\
pWL3EC & $12.6-\mathrm{kb}$, phaEC $C_{\text {Hme }}$ and its native promoter & {$[6]$} \\
pWL3EC1 & $12.5-\mathrm{kb}$, derivative of pWL3E, phaC1 insert & This study \\
pWL3EC2 & $12.2-\mathrm{kb}$, derivative of pWL3E, phaC2 insert & This study \\
pWL3EC3 & $12.9-\mathrm{kb}$, derivative of pWL3E, phaC3 insert & This study \\
\hline
\end{tabular}

Primers for RT-PCR (5'-3' sequence)

$\begin{array}{ll}\text { phaC-RT-F } & \text { GTACATTCTCGACCTCCA } \\ \text { phaC-RT-R } & \text { AGGAACGTTCCAAACGC } \\ \text { phaC1-RT-F } & \text { ATGACCATGAACCCGTT } \\ \text { phaC1-RT-R } & \text { CGATGTACCGATTCACGT } \\ \text { phaC2-RT-F } & \text { ATGTTCCCTAACCTCGTC } \\ \text { phaC2-RT-R } & \text { GTCCGCCTCAGTTCGTTC } \\ \text { phaC3-RT-F } & \text { ATGACAGATGATGGGCGT } \\ \text { phaC3-RT-R } & \text { CGATGTAGCGATTGACGT }\end{array}$

Primers for expression $\left(5^{\prime}-3^{\prime}\right.$ sequence $\left.{ }^{a}\right)$

\begin{tabular}{ll} 
phaC1-BamHI-F & ATAGGATCCATGACCATGAACCCGTTT \\
phaC1-Kpnl-R & AGCGGTACCTTAGCTGGTGCGTTGTCT \\
phaC2-BamHI-F & ATAGGATCCATGTTCCCTAACCTCGTC \\
phaC2-Kpnl-R & AGCGGTACCTTATCCGTTGGTGACGC \\
phaC3-BamHI-F & ATAGGATCCATGACAGATGATGGGCGT \\
phaC3-Kpnl-R & AGCGGTACCTCAGTCATCTTCTGTACC \\
\hline
\end{tabular}

${ }^{\mathrm{a}}$ Sequences representing the restriction sites are underlined.

respectively. To compare the PHBV synthesized by the four PHA synthases with distinct PhaC subunits, Har. hispanica PHB-1/pWL3EC [6] harboring the phaEC $C_{\mathrm{Hme}}$ genes of $H f x$. mediterranei was also employed.

Table 2 summarized the results of PHA accumulation in Har. hispanica and its five transformants. Compared with the wild-type strain, there was no significant difference in final cell yield as evaluated by cell dry weight (CDW). The expression of $\mathrm{PhaE}_{\mathrm{Hme}}$ alone or coexpression with PhaC2 did not lead to the synthesis of any
PHA. However, the other three $\mathrm{PhaC}$ proteins $\left(\mathrm{PhaC}_{\mathrm{Hme}}, \mathrm{PhaC} 1\right.$ and $\left.\mathrm{PhaC} 2\right)$ were functional during PHBV polymerization when excess glucose was supplied. PHBV content accumulated in recombinant PHB-1 strains harboring $\mathrm{PhaEC}_{\mathrm{Hme}}(17.33 \%)$ or PhaEC1 (15.61\%) was a little higher than that of wild type $(12.26 \%)$, and resulted in a much higher PHBV concentration of $0.99 \mathrm{~g} / \mathrm{l}$ or $0.94 \mathrm{~g} / \mathrm{l}$, respectively, as compared to $0.58 \mathrm{~g} / \mathrm{l}$ in wild type. In the case of PhaC3, the PHBV content $(10.02 \%)$ and concentration $(0.53 \mathrm{~g} / \mathrm{l})$ were

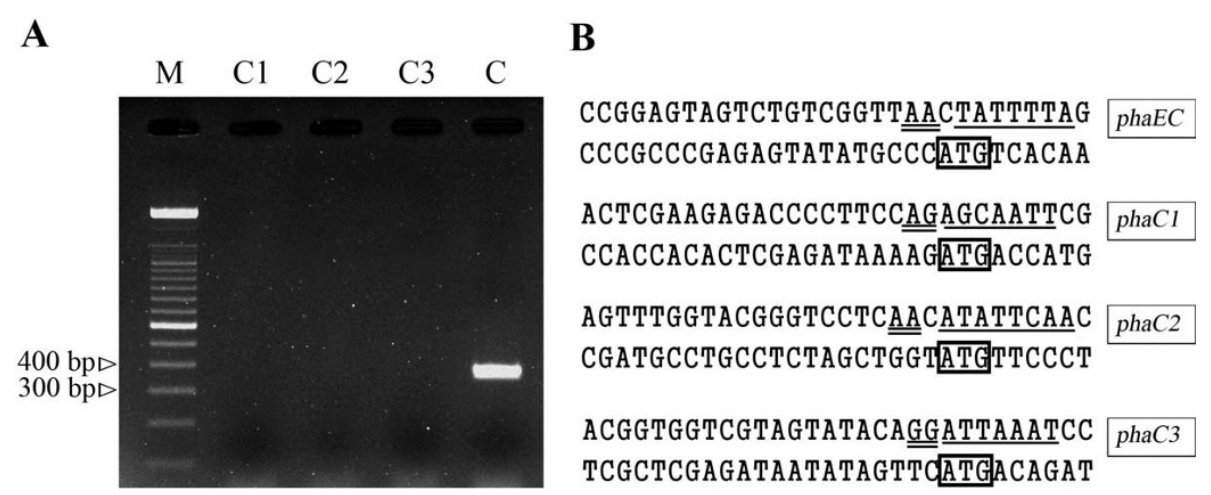

Figure 3 Transcriptional analysis of four phaC genes. (A) RT-PCR products of four phaC genes. Lane C1: phaC1 (391 bp); lane C2: phaC2 (408 bp); lane C3: phaC3 (412 bp); lane C: phaC Hme $(382$ bp); lane M: 100 bp DNA marker. (B) Promoter regions of the phaC genes. The putative TATA-box (single underlined), BRE (double underlined), and the translational start codons (boxed) of phaE $\mathrm{Hme}_{\mathrm{H}, \mathrm{phaC}}$, phaC2, and phaC3 are indicated. 
Table 2 PHA accumulation in Har. hispanica strains ${ }^{a}$

\begin{tabular}{cccccc}
\hline Har. hispanica Strain & $\begin{array}{c}\text { CDW } \\
(\mathbf{g} / \mathbf{L})\end{array}$ & $\begin{array}{c}\text { PHA content } \\
(\mathbf{w t} \%)\end{array}$ & $\begin{array}{c}\text { PHA concentration } \\
(\mathbf{g} / \mathbf{L})\end{array}$ & \multicolumn{2}{c}{ PHA composition (mol \%) } \\
\cline { 2 - 6 } Wild type & $4.70 \pm 0.23$ & $12.26 \pm 0.52$ & $0.58 \pm 0.03$ & $96.82 \pm 0.35$ & 3HB (C4) \\
PHB-1 (pWL3E) & $4.90 \pm 0.12$ & $\mathrm{ND}$ & $\mathrm{ND}$ & $\mathrm{ND}$ (C5) \\
PHB-1 (pWL3EC) & $5.27 \pm 0.61$ & $17.33 \pm 0.04$ & $0.99 \pm 0.11$ & $96.86 \pm 0.13$ & $3.14 \pm 0.13$ \\
PHB-1 (pWL3EC1) & $6.00 \pm 0.07$ & $15.61 \pm 0.06$ & $0.94 \pm 0.01$ & $95.94 \pm 0.50$ & $4.06 \pm 0.03$ \\
PHB-1 (pWL3EC2) & $5.58 \pm 0.21$ & $\mathrm{ND}$ & $\mathrm{ND}$ & $\mathrm{ND}$ & $\mathrm{ND}$ \\
PHB-1 (pWL3EC3) & $5.31 \pm 0.14$ & $10.02 \pm 1.06$ & $0.53 \pm 0.07$ & $98.05 \pm 0.20$ & $1.95 \pm 0.20$ \\
\hline
\end{tabular}

${ }^{a}$ The cells were cultured in MG medium at $37^{\circ} \mathrm{C}$ for $96 \mathrm{~h}$. For each strain, three samples were cultured and analyzed separately at the same time. Data are shown as the mean \pm standard deviation; $n=3$. ND, not detectable.

similar with that of wild type. Compared to that of wild type, the $3 \mathrm{HV}$ monomer composition of PHBV was a little higher for PhaEC1 (4.06 mol \%), a little lower for PhaEC3 (1.95 mol \%), and equivalent for $\mathrm{PhaEC}_{\mathrm{Hme}}$ (3.14 mol \%). In short, three types of PHBV with altered $3 \mathrm{HV}$ fractions had been synthesized by the Har. hispanica $\mathrm{PHB}-1$ recombinants with $p h a E_{\mathrm{Hme}}$ and different phaC genes.

\section{Properties of PHBV synthesized by different PhaC subunits}

A $400 \mathrm{MHz}{ }^{1} \mathrm{H}$ NMR study showed that the monomer composition of PHBV produced by the wild-type strain of Har. hispanica and its recombinants (data not shown) was in agreement with that determined by the GC analysis (Table 2). Table 3 listed the molecular weights and thermal properties of the accumulated PHBV in the recombinant strains of Har. hispanica PHB-1. The number-average molecular weight $\left(M_{\mathrm{n}}\right)$ and polydispersity $\left(M_{\mathrm{w}} / M_{\mathrm{n}}\right)$ of the polymers were in the range of 22.7-46.5 $\times 10^{4} \mathrm{Da}$ and of $1.83-2.16$, respectively. The PHBV synthesized by PhaEC3 had the highest $\mathrm{M}_{\mathrm{w}}$ of $91.0 \times 10^{4} \mathrm{Da}$, two times of that of the PHBV

Table 3 Molecular weights and thermal properties of PHBV synthesized by three PHA synthases with different PhaC subunits

\begin{tabular}{|c|c|c|c|c|c|c|c|}
\hline \multirow[t]{2}{*}{ PHBV type ${ }^{a}$} & \multicolumn{3}{|c|}{ Molecular weight $^{b}$} & \multicolumn{4}{|c|}{$\begin{array}{l}\text { Thermal properties } \\
\left({ }^{\circ} \mathrm{C}\right){ }^{\mathrm{C}}\end{array}$} \\
\hline & $\begin{array}{c}M_{\mathrm{w}}\left(10^{4}\right. \\
\mathrm{Da})\end{array}$ & $\begin{array}{c}M_{\mathrm{n}}\left(10^{4}\right. \\
\mathrm{Da})\end{array}$ & $\begin{array}{c}M_{\mathrm{w}} / \\
M_{\mathrm{n}}\end{array}$ & $T_{\mathrm{d}}$ & $T_{\mathrm{g}}$ & $T_{\mathrm{c}}$ & $\overline{T_{\mathrm{m}}}$ \\
\hline $\begin{array}{l}\text { PhaEC }_{H m e}- \\
\text { PHBV }\end{array}$ & 34.4 & 62.8 & 1.83 & 252 & 2.3 & 54.6 & 163.2 \\
\hline PhaEC1-PHBV & 49.1 & 22.7 & 2.16 & 257 & -0.1 & 50.5 & 160.3 \\
\hline PhaEC3-PHBV & 91.0 & 46.5 & 1.96 & 255 & 5.7 & ND & 162.3 \\
\hline
\end{tabular}

a PHBV synthesized by different PHA synthases (PhaEC ${ }_{\mathrm{Hme}}$, PhaEC1, or PhaEC3) in Har. hispanica; ${ }^{\mathrm{b}} M_{\mathrm{w}}$, weight-average molecular weight; $M_{\mathrm{n}}$, number-average molecular weight; ${ }^{\mathrm{C}} T_{\mathrm{d}}$, temperature at $5 \%$ weight loss determined by TGA; $T_{\mathrm{g}}$ glass-transition temperature; $T_{c}$, cold crystallization temperature; $T_{\mathrm{m}}$, melting temperature; ND, not detectable. The experiments were repeated three times and the results of one representative experiment were shown. produced by PhaEC1, but the PHBV production was lower (Table 2).

DSC analysis showed that the glass-transition temperatures $\left(T_{\mathrm{g}}\right)$ of the $\mathrm{PHBV}$ samples were $2.3^{\circ} \mathrm{C}$ (PhaEC $\mathrm{Hme}_{\mathrm{Hm}}$-PHB), $-0.1^{\circ} \mathrm{C}$ (PhaEC1-PHBV), and $5.7^{\circ} \mathrm{C}$ (PhaEC3-PHBV). This result was consistent with the $3 \mathrm{HV}$ monomer composition of the three types of PHBV; a larger amount of $3 \mathrm{HV}$ incorporated into PHBV could lead to lower $T_{\mathrm{g}}$. A similar melting temperature $\left(T_{\mathrm{m}}\right)$ was observed for all three PHBV samples, of which the PHBV with the lowest $T_{\mathrm{m}}$ synthesized by PhaEC1 could facilitate its processing ability. Compared with the other two polymers, no cold crystallization temperature $\left(T_{\mathrm{c}}\right)$ was detected for the PHBV accumulated by PhaEC3. This result demonstrated that this polymer (with a high $3 \mathrm{HB}$ fraction) possessed a fast crystallization speed, which could also provide some advantages in processing.

Thermal stability plays an important role in polymer melt processing. This characteristic of the three types of PHBV was determined by TGA (Table 3 and Figure 4). The temperature at $5 \%$ weight loss $\left(T_{\mathrm{d}}\right)$ was used to evaluate their polymer thermal stability. The $T_{\mathrm{d}}$ of the three polymers ranged from $252^{\circ} \mathrm{C}$ to $257^{\circ} \mathrm{C}$. Our results showed that the three types of $\mathrm{PHBV}$, with different monomer composition, changed obviously in their thermal properties.

\section{Discussion}

Multiple phaC genes in a same genome have been found in the domain of Bacteria. The complete genome sequence of Ralstonia eutropha has revealed the existence of a second phaC gene, which is not clustered with the phaCAB operon, and its function has not been clarified [25]. Additionally, Pseudomonas strains synthesizing medium-chain-length PHAs (mcl-PHAs) usually have two different phaC genes, which are separated by a phaZ gene that encodes an intracellular PHA depolymerase [1]. To date, the haloarchaeon $H f x$. mediterranei has been found to possess the largest number of phaC genes. Compared with the other PhaC subunits from haloarchaeal strains, the three newly identified PhaCs 


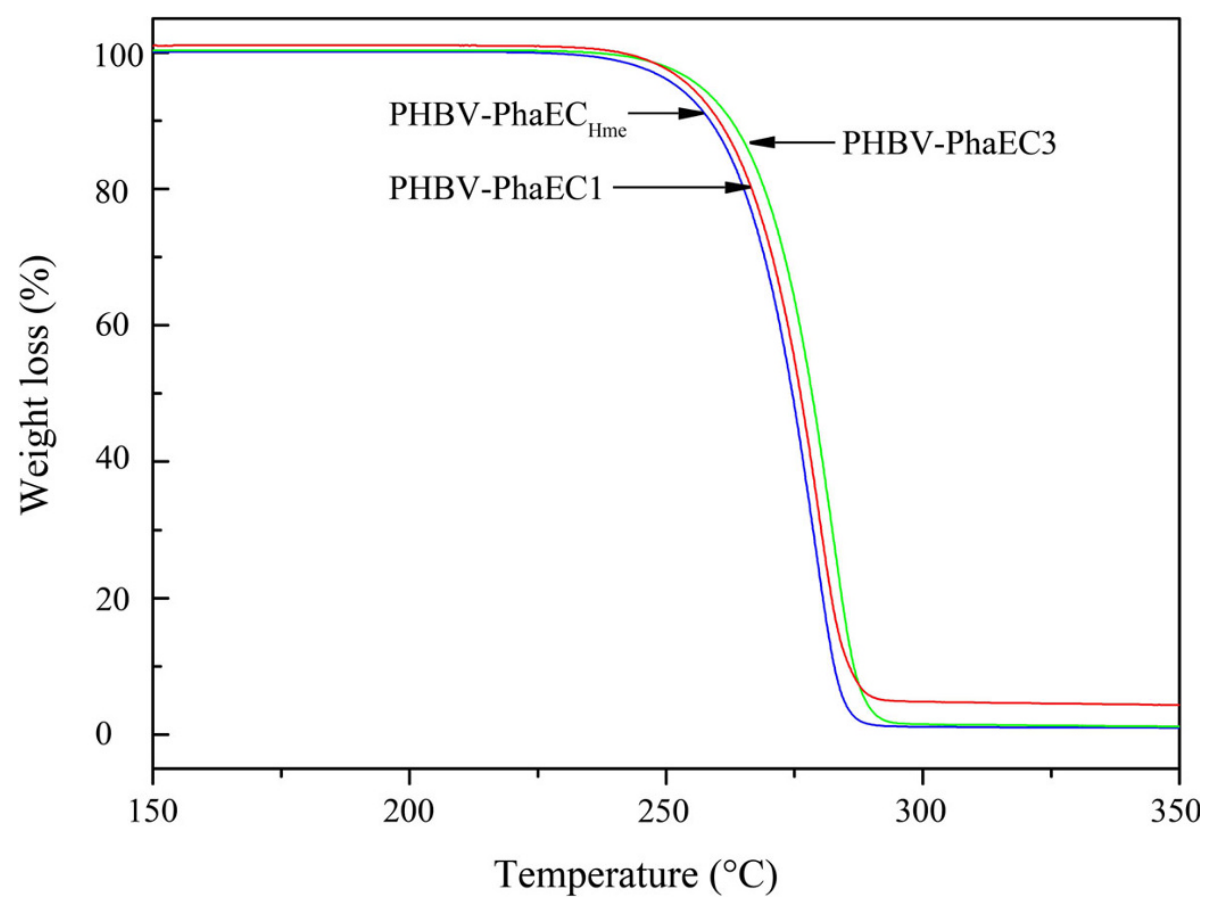

Figure 4 Thermogravimetric analysis (TGA) of PHBV polymers produced by Har. hispanica PHB-1 recombinants harboring PhaEC $\mathrm{Hme}_{\mathrm{H}}$ PhaEC1, or PhaEC3

showed less homology with $\mathrm{PhaC}_{\mathrm{Hme}}$. A phylogenetic tree was constructed based on the four PhaCs from $H f x$. mediterranei and seven other PhaCs from Halobacteriaceae; 3 PhaC sequences from Thaumarchaeota were used as the outgroup (Figure 5). The PhaCs from Halobacteriaceae and those from Thaumarchaeota clustered separately. $\mathrm{PhaC}_{\mathrm{Hme}}$ was more closely related to the PhaCs from other haloarchaea than to its three paralogs (Figure 5). It seemed likely that phaC1, phaC2 and phaC3 were not directly acquired from a common source of $\mathrm{PhaC}_{\mathrm{Hme}}$, but possibly by horizontal DNA transfer from other sources during evolution.

Of the four paralogs in Hfx. mediterranei, only $p h a C_{\text {Hme }}$ was found to be transcribed under PHA-accumulating conditions by RT-PCR assays (Figure 3A). However, the putative promoters (including the TATA box and BRE element) of the other three phaC genes, were also detected (Figure 3B). As both phaC1 and phaC3 encoded functional proteins, it is conceivable that these silent genes in $H f x$. mediterranei might be activated under specific ecological conditions to increase the environmental distribution of this haloarchaeon.

Regardless of the conserved catalytic triad residues (Cys-Asp-His) in the primary sequence, PhaC2 was found to have no PHA polymerization activity. This might be the result of the truncated $C$ terminus, which has been shown to be indispensable for PHA synthase enzyme activity [6]. However, a recombinant PhaC2 containing the $\mathrm{C}$ terminus from $\mathrm{PhaC} 3$ does not have the ability to synthesize PHAs (data not shown). Alternatively, less conserved motifs such as the "Ala" residue in the "Lipase box-like" sequence or the lack of the conserved motif of PhaC might lead to its inactivity.

The incorporation of $3 \mathrm{HV}$ into $\mathrm{PHB}$ could improve material flexibility. Three types of PHBV with different monomer compositions were produced from the Har. hispanica PHB-1 recombinants, and the type of PHBV was supposed to be determined by the different substrate specificities of $\mathrm{PhaC}_{\mathrm{Hme}}$, PhaC1, and PhaC3. $H f x$. mediterranei has been found to be the best PHBV producer in the family Halobacteriaceae, and can accumulate the highest PHBV content $(>60 \%)$ with the highest $3 \mathrm{HV}$ fraction from many unrelated cheap carbon sources [7,20,21]. Compared with Har. hispanica, Hfx. mediterranei has a stronger ability to provide more $3 \mathrm{HB}-\mathrm{CoA}$ and $3 \mathrm{HV}-\mathrm{CoA}$ monomers for PHA synthase. Therefore, the use of these phaC genes in $H f x$. mediterranei PHBV-1 to produce PHBV with higher 3HV fractions and different properties would have much more promise, which could extend the applications of PHAs in the future.

\section{Conclusions}

In this study, we mined three silent phaC genes (phaC1, phaC2, and phaC3) from $H f x$. mediterranei, which encoded potential PhaCs subunits of PHA synthase. In 


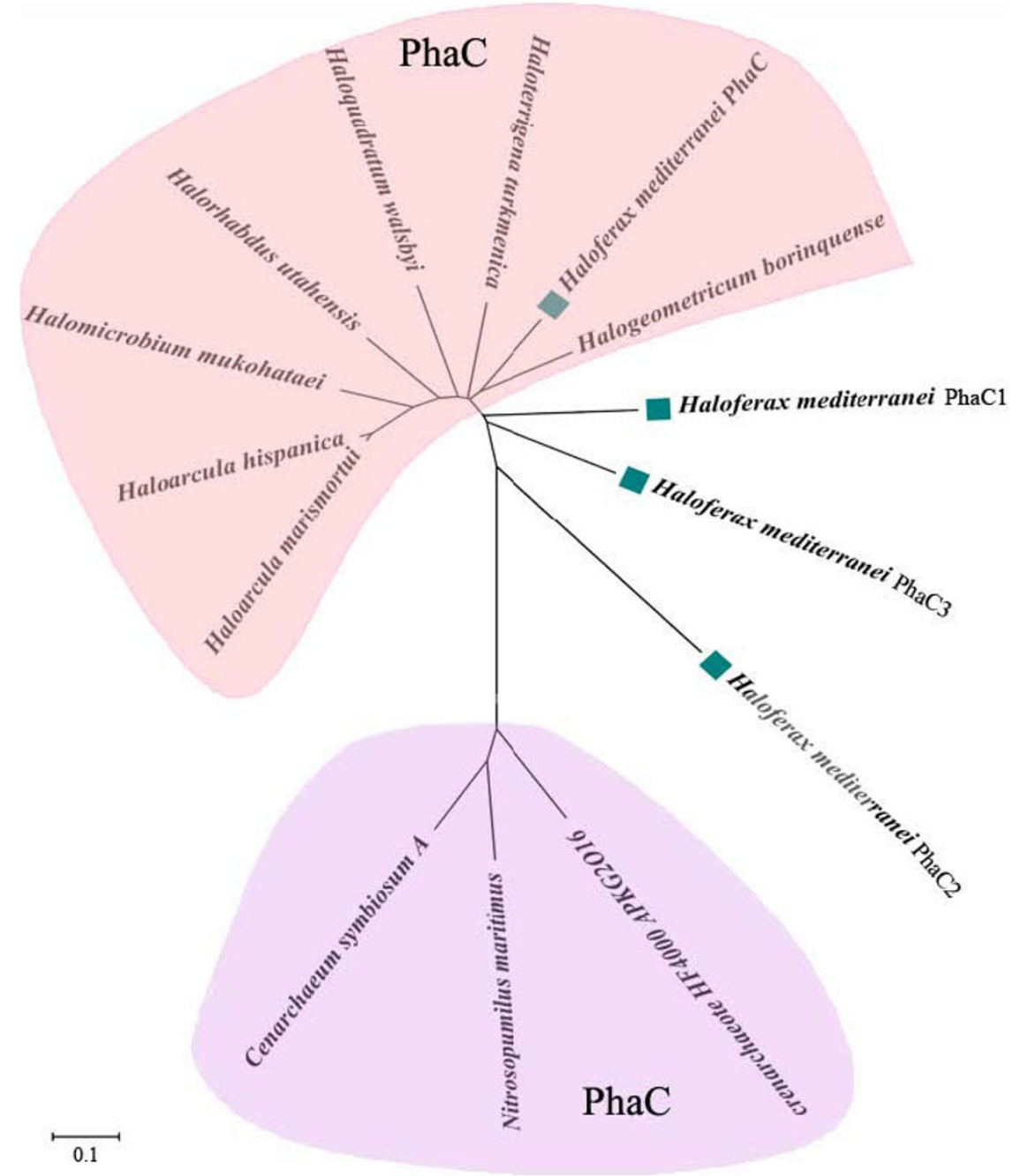

Figure 5 Phylogenetic analysis of PhaCs from the domain of Archaea. The tree was constructed using the neighbor-joining algorithm with MEGA software version 4.0. The numbers next to the nodes indicate the bootstrap values based on 1000 replications (expressed as percentages). Scale bar $=0.1$ substitution per site. GenBank accession numbers of PhaCs were as follows: Haloarcula marismortui, YP_137339; Hloarcula hispanica, ABV71394; Halomicrobium mukohataei, YP_003176827; Halorhabdus utahensis, YP_003131063; Haloquadratum walsbyi, YP_658052; Haloterrigena turkmenica, YP_003404006; Halogeometricum borinquense, ZP_03999959; Crenarchaeote HF4000_APKG2016, ABZ08353; Nitrosopumilus maritimus, YP_001582325; Cenarchaeum symbiosum, YP_876256; Haloferax mediterranei, phaC Hme-EU374220, phaC1-HM116246, phaC2-HM116247, phaC3-HM116248. The four PhaC of Hfx. mediterranei are marked with green square.

Har. hispanica PHB-1, the heterologous coexpression of pha $E_{\mathrm{Hme}}$ with phaC $C_{\mathrm{Hme}}$, phaC1, or phaC3 could lead to the accumulation of $\mathrm{PHBV}$ polymers with different molecular weights and thermal properties from excess glucose. Therefore, we concluded that genetic engineering of these phaC genes in Hfx. mediterranei would produce PHBV with desirable material properties, at lower production costs.

\section{Methods}

\section{Strains, plasmids, and growth conditions}

The plasmids used in this study were listed in Table 1. All of the plasmids were derivatives of pWL3E [6].
These plasmids were first constructed in Escherichia coli JM109 and then introduced into Har. hispanica PHB-1, a phaEC-deleted strain [13], using the polyethylene glycol-mediated transformation method [26].

E. coli JM109 was cultivated in Luria-Bertani (LB) medium at $37^{\circ} \mathrm{C}$ [27]. When needed, ampicillin (100 $\mathrm{mg} / \mathrm{l}$ ) was added to the medium. Har. hispanica and Hfx. mediterranei were grown in AS-168 medium. For growth on plates, the media described above were supplemented with $1.2 \%(\mathrm{w} / \mathrm{v})$ agar. For PHA accumulation, the haloarchaeal strains were transferred from AS-168 medium to MG medium and cultivated as previously described [6]. When required, mevinolin was added to a 
final concentration of $5 \mathrm{mg} / \mathrm{l}$ for $H$. hispanica recombinants. All of the strains were cultured at $37^{\circ} \mathrm{C}$.

\section{Primers and plasmid construction}

All of the primers used in this study were summarized in Table 1. To determine the capabilities of PhaC1, PhaC2, and PhaC3 to synthesize PHAs, three expression plasmids were constructed. The expression vectors were derived from $\mathrm{pWL} 3 \mathrm{E}$, which contained the phaE $E_{\mathrm{Hme}}$ gene of $H f x$. mediterranei with its native promoter [6]. The coding sequences of phaC1, phaC2, and phaC3 were amplified from $H f x$. mediterranei by PCR with primer pairs phaC1-BamHI-F/phaC1-KpnI-R, phaC2BamHI-F/phaC2-KpnI-R, and phaC3-BamHI-F/phaC3KpnI-R, respectively. The purified PCR products (phaC1, $1.3 \mathrm{~kb}$; phaC2, $1.1 \mathrm{~kb}$; phaC3, $1.7 \mathrm{~kb}$ ) were digested with $B a m \mathrm{HI}$ and $K p n \mathrm{I}$ and inserted into BamHI and KpnI-treated pWL3E. The resulting expression plasmids pWL3EC1, pWL3EC2, and pWL3EC3 were confirmed by DNA sequencing and individually introduced into Har. hispanica PHB-1. The haloarchaeal recombinants harboring pWL3EC [6], pWL3EC1, pWL3EC2, and pWL3EC3 were subjected to PHA accumulation analysis in which Har. hispanica PHB-1 harboring pWL3E and the wild-type strain were used as the negative and positive control, respectively.

\section{Isolation of total RNA and reverse transcription PCR (RT- PCR)}

Total RNA of $H f x$. mediterranei was extracted using TRIzol reagent (Invitrogen) from a logarithmic-phase culture grown in MG medium. Four specific primer pairs, phaC-RT-F/phaC-RT-F, phaC1-RT-F/phaC1-RTF, phaC2-RT-F/phaC2-RT-F, and phaC3-RT-F/phaC3$\mathrm{RT}-\mathrm{F}$, were designed for the pha $C_{\mathrm{Hme}}$, phaC1, phaC2, and phaC3 genes, respectively (Table 1 ). Prior to RTPCR, the specificity of these primers were confirmed by sequencing of the corresponding PCR products using the genomic DNA of $H f x$. mediterranei as template. For RT-PCR, the RNA sample was digested with RNase-free RQ1 DNase (Promega) overnight to eliminate any DNA contamination, which was subsequently confirmed by a control PCR without prior reverse transcription as previously described [28]. The DNA-free RNA sample was then used as the template for RT-PCR using the OneStep RT-PCR Kit (Qiagen) according to the manufacturer's instruction. The PCR products were electrophoresed in a $1 \%$ agarose gel.

\section{Measurement of PHA content and composition}

Scl-PHAs were qualitatively and quantitatively analyzed by gas chromatography (GC) with the Agilent GC-6820 as described previously $[6,13]$. Benzonic acid was used as an internal standard to calculate the amount of
PHAs. PHBV (Sigma) was used as the standard for peak identification. The average results of three parallel experiments were recorded.

\section{Extraction and purification of PHAs}

After fermentation, the liquid cultures were harvested by centrifugation at $9000 \mathrm{rpm}$ for $10 \mathrm{~min}$. The cell pellets were resuspended in distilled water containing $0.1 \% \operatorname{SDS}(\mathrm{w} / \mathrm{v})$ and agitated for $20 \mathrm{~min}$. The suspension was centrifuged at $5000 \mathrm{rpm}$ for $5 \mathrm{~min}$ and yielded whitish, compact sediment. The sediment was resuspended and washed twice with $0.1 \%$ SDS solution $(\mathrm{w} / \mathrm{v})$ and distilled water, respectively. The resulting paste was dried in an oven at $70^{\circ} \mathrm{C}$ for $2 \mathrm{~h}$ for further purification. The extracted PHAs were treated with hot chloroform at $90^{\circ} \mathrm{C}$ for $4 \mathrm{~h}$, followed by precipitation with 15 volume of pre-chilled ethanol. To obtain pure product, the precipitate was then redissolved in chloroform and the process was repeated. The collected PHAs were dried at $40^{\circ} \mathrm{C}$ for $24 \mathrm{~h}$ to remove all residual solvent.

\section{Molecular weight determination of PHBV}

The molecular weight of PHBV was estimated via gel permeation chromatography (GPC) with a Waters 1515 system at $30^{\circ} \mathrm{C}$ using a Waters 1525 pump equipped with a four Styragel column series (Styragel HR, $5 \mu \mathrm{m}$ ). A differential refractive index detector and a UV detector were both employed. Chloroform was used as the elution solvent at a flow rate of $1.0 \mathrm{ml} / \mathrm{min}$. The concentration of the samples was $2 \mathrm{mg} / \mathrm{ml}$ and the injection volume was $50 \mu \mathrm{l}$. The calibration curve was generated with polystyrene standards (Sigma).

\section{Characterization of PHBV thermal properties}

Differential scanning calorimetry (DSC) was performed using a TA Instruments Q100 with an autocool accessory calibrated with indium to characterize thermal transitions of the polymers. The following protocol was used for each sample. A 3-5 mg sample in an aluminum pan was cooled from room temperature to $-60^{\circ} \mathrm{C}$ by the autocool accessory. The pan was heated from $-60^{\circ} \mathrm{C}$ to $180^{\circ} \mathrm{C}$ at a rate of $10^{\circ} \mathrm{C} / \mathrm{min}$, isothermally maintained at $180^{\circ} \mathrm{C}$ for $5 \mathrm{~min}$, quenched to $-60^{\circ} \mathrm{C}$, and reheated from $-60^{\circ} \mathrm{C}$ to $180^{\circ} \mathrm{C}$ at $10^{\circ} \mathrm{C} / \mathrm{min}$ under a nitrogen flow rate of $50 \mathrm{ml} / \mathrm{min}$. Data were collected during the second heating run.

Thermal stability of the PHA polymer was determined via thermogravimetric analysis using a Q50 thermogravimetric analyzer (TGA) (TA Instruments). The procedures used were as the same as those described by Xie et al. [29]. Briefly, samples were analyzed at a heating rate of $10^{\circ} \mathrm{C} / \mathrm{min}$ under a nitrogen atmosphere with a $\mathrm{N} 2$ flow rate of $60 \mathrm{ml} / \mathrm{min}$. 


\section{Phylogenetic tree construction}

The phylogenetic tree for $\mathrm{PhaC}$ was constructed using the neighbor-joining method [30] with Molecular Evolutionary Genetics Analysis (MEGA) software version 4.0 [31]. The topology of the phylogenetic tree was evaluated by bootstrap analysis on the basis of 1000 replications [32].

\section{Nucleotide and protein sequence analysis}

Sequences of the PhaC proteins from Hfx. mediterranei were deposited in GenBank under the accession numbers pha $C_{\mathrm{Hme}}$-EU374220, phaC1-HM116246, phaC2HM116247, and phaC3-HM116248. These PhaC sequences were analyzed using the GeneDoc program (http://www.nrbsc.org/gfx/genedoc/index.html).

\section{Acknowledgements}

This work was supported by grants from the National Natural Science Foundation of China (30621005, 30830004, and 30925001).

\section{Author details}

${ }^{1}$ State Key Laboratory of Microbial Resources, Institute of Microbiology, Chinese Academy of Sciences, No.1 West Beichen Road, Chaoyang District, Beijing 100101, China. ${ }^{2}$ Multidisciplinary Research Center, Shantou University, China.

\section{Authors' contributions}

$J H(H a n)$ performed most of the experimental work and drafted the manuscript. ML extracted the total RNA and was involved in gene expression analysis. $\mathrm{JH}(\mathrm{Hou})$ and $\mathrm{JZ}$ extracted and purified the polymers. LW was involved in copolymer characterization. HX was involved in experimental design, data analysis, and finalized the manuscript. All the authors have read and approved the final manuscript.

\section{Competing interests}

The authors declare that they have no competing interests.

Received: 4 May 2010 Accepted: 20 August 2010

Published: 20 August 2010

\section{References}

1. Rehm BH: Polyester synthases: natural catalysts for plastics. Biochemical Journal 2003, 376(1):15-33.

2. Marchessault RH: Tender morsels for bacteria: Recent developments in microbial polyesters. Trends Polym Sci 1996, 4:163-168.

3. Salehizadeh H, Van Loosdrecht MC: Production of polyhydroxyalkanoates by mixed culture: recent trends and biotechnological importance. Biotechnology Advances 2004, 22(3):261-279.

4. Aldor IS, Keasling JD: Process design for microbial plastic factories: metabolic engineering of polyhydroxyalkanoates. Current Opinion in Biotechnology 2003, 14(5):475-483.

5. Lee SY: Plastic bacteria? Progress and prospects for polyhydroxyalkanoate production in bacteria. Trends in Biotechnology 1996, 14:431-438.

6. Lu Q, Han J, Zhou L, Zhou J, Xiang H: Genetic and biochemical characterization of the poly(3-hydroxybutyrate-co-3-hydroxyvalerate) synthase in Haloferax mediterranei. Journal of Bacteriology 2008, 190(12):4173-4180

7. Koller M, Hesse P, Bona R, Kutschera C, Atlić A, Braunegg G: Potential of various archae- and eubacterial strains as industrial polyhydroxyalkanoate producers from whey. Macromolecular Bioscience 2007, 7(2):218-226.

8. Lillo JG, Rodriguez-Valera F: Effects of culture conditions on poly( $\beta$ hydroxybutyric acid) production by Haloferax mediterranei. Applied and Environmental Microbiology 1990, 56(8):2517-2521.
9. Hezayen FF, Rehm BH, Eberhardt R, Steinbüchel A: Polymer production by two newly isolated extremely halophilic archaea: application of a novel corrosion-resistant bioreactor. Applied Microbiology and Biotechnology 2000, 54(3):319-325.

10. Wainø M, Tindall BJ, Ingvorsen K: Halorhabdus utahensis gen. nov., sp. nov., an aerobic, extremely halophilic member of the Archaea from Great Salt Lake, Utah. International Journal of Systematic and Evolutionary Microbiology 2000, 50:183-190

11. Hezayen FF, Tindall BJ, Steinbüchel A, Rehm BH: Characterization of a novel halophilic archaeon, Halobiforma haloterrestris gen. nov., sp. nov., and transfer of Natronobacterium nitratireducens to Halobiforma nitratireducens comb. nov. International Journal of Systematic and Evolutionary Microbiology 2002, 52(6):2271-2280.

12. Legault BA, Lopez-Lopez A, Alba-Casado JC, Doolittle WF, Bolhuis H, Rodriguez-Valera F, Papke RT: Environmental genomics of "Haloquadratum walsbyi" in a saltern crystallizer indicates a large pool of accessory genes in an otherwise coherent species. BMC Genomics 2006, 7:171.

13. Han J, Lu Q, Zhou L, Zhou J, Xiang H: Molecular characterization of the phaEC $\mathrm{Hm}_{\mathrm{m}}$ genes, required for biosynthesis of poly(3-hydroxybutyrate) in the extremely halophilic archaeon Haloarcula marismortui. Applied and Environmental Microbiology 2007, 73(19):6058-6065.

14. Romano I, Poli A, Finore I, Huertas FJ, Gambacorta A, Pelliccione S, Nicolaus G, Lama L, Nicolaus B: Haloterrigena hispanica sp. nov., an extremely halophilic archaeon from Fuente de Piedra, southern Spain. International Journal of Systematic and Evolutionary Microbiology 2007, 57:1499-1503.

15. Antunes A, Taborda M, Huber R, Moissl C, Nobre MF, da Costa MS: Halorhabdus tiamatea sp. nov., a non-pigmented, extremely halophilic archaeon from a deep-sea, hypersaline anoxic basin of the Red Sea, and emended description of the genus Halorhabdus. International Journal of Systematic and Evolutionary Microbiology 2008, 58:215-220.

16. Hezayen FF, Gutierrez CM, Steinbüchel A, Tindall BJ, Rehm BH: Halopiger aswanensis sp. nov., a polymer-producing, extremely halophilic archaeon isolated from hypersaline soil. International Journal of Systematic and Evolutionary Microbiology 2009, 60:633-637

17. Bloemnergen SHDA, Harmer GK, Bluhn TL, Marchessault RH: Studies of composition and crystallinity of bacterial poly ( $\beta$-hydroxybutyrate-co$\beta$-hydroxyvalerate). Macromolecules 1986, 19:2865-2871.

18. Mergaert J, Webb A, Anderson C, Wouters A, Swings J: Microbial degradation of poly(3-hydroxybutyrate) and poly(3-hydroxybutyrate-co3-hydroxyvalerate) in soils. Applied and Environmental Microbiology 1993, 59(10):3233-3238.

19. Koning GD, Mergaert J: Physical properties of bacterial poly((R)-3hydroxyalkanoates). Canadian Journal of Microbiology 1995, 41(Suppl 1):303-309.

20. Don TM, Chen CW, Chan TH: Preparation and characterization of poly (hydroxyalkanoate) from the fermentation of Haloferax mediterranei. Journal of Biomaterials Science 2006, 17(12):1425-1438.

21. Huang TY, Duan K, Huang SY, Chen CW: Production of polyhydroxyalkanoates from inexpensive extruded rice bran and starch by Haloferax mediterranei. Journal of Industrial Microbiology and Biotechnology 2006, 33(8):701-706.

22. Stubbe J, Tian J: Polyhydroxyalkanoate (PHA) hemeostasis: the role of PHA synthase. Natural Product Reports 2003, 20(5):445-457.

23. Hai T, Lange D, Rabus R, Steinbüchel A: Polyhydroxyalkanoate (PHA) accumulation in sulfate-reducing bacteria and identification of a Class III PHA synthase (PhaEC) in Desulfococcus multivorans. Applied and Environmental Microbiology 2004, 70(8):4440-4448.

24. López-garcia P, Abad JP, Smith C, Amils R: Genomic organization of the halophilic archaeon Haloferax mediterranei: physical map of the chromosome. Nucleic Acids Research 1992, 20(10):2459-2464.

25. Pohlmann A, Fricke WF, Reinecke F, Kusian B, Liesegang H, Cramm R, Eitinger T, Ewering C, Pötter M, Schwartz E, et al: Genome sequence of the bioplastic-producing "Knallgas" bacterium Ralstonia eutropha H16. Nature Biotechnology 2006, 24(10):1257-1262

26. Cline SW, Lam WL, Charlebois RL, Schalkwyk LC, Doolittle WF: Transformation methods for halophilic archaebacteria. Canadian Journal of Microbiology 1989, 35(1):148-152.

27. Sambrook J, Fritsch EF, Maniatis T: Molecular Cloning: A Laboratory Manual. Cold Spring Harbor Laboratory Press, 21989. 
28. Han J, Lu Q, Zhou L, Liu H, Xiang H: Identification of the polyhydroxyalkanoate (PHA)-specific acetoacetyl-CoA reductase from multiple FabG paralogs in Haloarcula hispanica and reconstruction of PHA biosynthetic pathway in Haloferax volcanii. Applied and Environmental Microbiology 2009, 75:6168-6175.

29. Xie WP, Chen GQ: Production and characterization of terpolyester poly(3hydroxybutyrate-co-4-hydroxybutyrate-co-3-hydroxyhexanoate) by recombinant Aeromonas hydrophila 4AK4 harboring genes phaPCJ. Biochemical Engineering Journal 2008, 38(3):384-389.

30. Saitou N, Nei M: The neighbor-joining method: a new method for reconstructing phylogenetic trees. Molecular Biology and Evolution 1987, 4(4):406-425.

31. Tamura K, Dudley J, Nei M, Kumar S: MEGA4: Molecular evolutionary genetics analysis (MEGA) software version 4.0. Molecular Biology and Evolution 2007, 24(8):1596-1599.

32. Felsenstein J: Confidence limits on phylogenies: An approach using the bootstrap. Evolution 1985, 39:783-791.

doi:10.1186/1746-1448-6-9

Cite this article as: Han et al: Comparison of four phaC genes from Haloferax mediterranei and their function in different PHBV copolymer biosyntheses in Haloarcula hispanica. Saline Systems 2010 6:9.

\section{Submit your next manuscript to BioMed Central} and take full advantage of:

- Convenient online submission

- Thorough peer review

- No space constraints or color figure charges

- Immediate publication on acceptance

- Inclusion in PubMed, CAS, Scopus and Google Scholar

- Research which is freely available for redistribution

Submit your manuscript at www.biomedcentral.com/submit 